\title{
AMEAÇAS DA JUDICIALIZAÇÃO DA SAÚDE À INTEGRIDADE DO SISTEMA DE SAÚDE SEGUNDO A TEORIA DOS SISTEMAS SOCIAIS DE NIKLAS LUHMANN: UMA ANÁLISE A PARTIR DOS DADOS DO ESTADO DO RIO GRANDE DO SUL EM 2018
}

\section{THREATS OF THE JUDICIALIZATION OF HEALTH TO THE INTEGRITY OF THE HEALTH SYSTEM ACCORDING TO THE THEORY OF THE SOCIAL SYSTEMS OF NIKLAS LUHMANN: AN ANALYSIS FROM THE DATA OF THE STATE OF RIO GRANDE DO SUL IN 2018}

\section{Tarsila Rorato Crusius ${ }^{1}$ Mártin Perius Haeberlin²}

DOI: https://doi.org/10.37767/2591-3476(2020)13

\begin{abstract}
RESUMEN:
El estudio busca comprender cómo la judicialización puede comprometer la integridad del sistema de la salud, desde la perspectiva de la Teoría General de los Sistemas Sociales de Niklas Luhmann. Para esto, primero busca saber cómo las decisiones judiciales actúan como irritaciones para el sistema de la salud. Despues, analiza el impacto de estas decisiones en el sistema, basándose en datos de la judicialización en Rio Grande do Sul, en 2018. Finalmente, analiza los riesgos para la integridad del sistema de salud representado por la judicialización, así como las respuestas del sistema político brasileño. Se presume que las respuestas del sistema de la salud a las amenazas planteadas por la judicialización dependen de las respuestas de otros sistemas sociales. La metodología es exploratoria, con investigación bibliográfica. Se concluye que la capacidad de respuesta de los sistemas sociales a estas irritaciones definirá la posibilidad de mantener su integridad y diferenciación.
\end{abstract}

RESUMO:

O estudo busca compreender como a judicialização pode comprometer a integridade do sistema da saúde na perspectiva da Teoria Geral dos Sistemas Sociais de Niklas Luhmann. Para isso, primeiramente busca conhecer como decisões judiciais atuam como irritações ao sistema da saúde. Em um segundo momento, analisa o impacto destas de-

1 Mestranda em Direitos Humanos pela UniRitter - Laureate International Universities, com bolsa CAPES. Psicóloga, com especialização em Gestão do Trabalho e da Educação na Saúde. Contato: tarsila.crusius@gmail.com. ORCID iD: https://orcid.org/0000-0002-7081-5040.

2 Doutor em Direito (PUCRS). Pós-doutorando em Economia (UFRGS), com bolsa CAPES. Professor da Graduação em Direito e do Mestrado em Direitos Humanos da UniRitter - Laureate International Universities. Contato: martin_haeberlin@uniritter.edu.br. ORCID iD: https://orcid.org/0000-0003-3101-5419. 
cisões no sistema, a partir dos dados da judicialização em saúde no Estado do Rio Grande do Sul, em 2018. Por fim analisa os riscos à integridade do sistema da saúde representados pela judicialização, bem como as respostas estruturadas pelo sistema político brasileiro. Parte-se da hipótese de que as respostas do sistema da saúde às ameaças representadas pela judicialização da saúde dependem das respostas de outros sistemas sociais. A metodologia é de caráter exploratório, com realização de pesquisa bibliográfica. Conclui-se que a capacidade de resposta dos sistemas sociais a estas irritações definirá a possibilidade de manutenção de sua integridade e diferenciação.

\begin{abstract}
This study seeks to understand how judicialization may compromise the integrity of the health system from Niklas Luhmann's General Theory of Social Systems perspective. For this, it first seeks to know how judicial decisions act as irritations to the health system. Secondly, it analyzes the impact of these decisions on the system, based on data from health judicialization in the state of Rio Grande do Sul, in 2018. Finally, it analyzes the risks to the integrity of the health system represented by judicialization, as well as the responses structured by the Brazilian political system. It is hypothesized that health system responses to threats posed by judicialization depend on responses from other social systems. The methodology is exploratory, with bibliographical research. It is concluded that the responsiveness of social systems to these irritations will define the possibility of maintaining their integrity and differentiation.
\end{abstract}

PALABRAS CLAVE: Teoria General de los Sistemas Sociales de Niklas Luhmann; Derecho a la Salud; Judicialización Sanitaria; Sistema de la Salud.

PALAVRAS-CHAVE: Teoria Geral dos Sistemas Sociais de Niklas Luhmann; Direito à Saúde; Judicialização em Saúde; Sistema da Saúde.

KEY WORDS: Niklas Luhmann General Theory of Social Systems; Right to Health; Health Judicialization; Health System.

\title{
I. Introdução
}

Nos últimos anos, o crescimento do fenômeno da judicialização da saúde vem sendo objeto de preocupação e estudo por parte de gestores públicos, juristas e pesquisadores. Esta preocupação decorre não somente do grande impacto das decisões judiciais nos orçamentos da União, Estados e Municípios, como também da maneira como o fenômeno interfere com a própria lógica interna de funcionamento deste sistema.

A judicialização da saúde vem aportando novos desafios e complexidades aos sistemas sociais envolvidos. Se, por um lado, ela tem o potencial de gerar um quadro de corrupção sistêmica, por outro lado as respostas do conjunto de sistemas sociais às irritações/ perturbações provocadas pelas decisões judiciais podem ser geradoras de mudanças que produzam um aumento da diferenciação e, portanto, uma evolução sistêmica.

O presente estudo propõe-se a compreender de que maneira a judicialização pode comprometer a integridade do sistema da saúde a partir da abordagem da Teoria Geral 
dos Sistemas Sociais de Niklas Luhmann. Pretende-se, por meio dele, contribuir para uma melhor compreensão das ameaças representadas pela judicialização à integridade do sistema da saúde, bem como das formas por meio das quais os sistemas sociais vêm respondendo aos desafios da judicialização.

Para esse fim, busca conhecer, em um primeiro momento, a forma pela qual as decisões judiciais, oriundas do sistema do Direito, atuam como irritações/perturbações ao sistema de saúde. Em um segundo momento, busca compreender o impacto destas decisões no sistema da saúde a partir de dados referentes ao fornecimento judicial de medicamentos e demais tratamentos no Estado do Rio Grande do Sul. Por fim, os riscos à integridade do sistema da saúde, representados pela judicialização, são discutidos a partir dos dados analisados, assim como a partir de recentes respostas do sistema político aos desafios apresentados, com consequências no sistema do direito.

A hipótese trabalhada é a de que o sistema da saúde poderá responder às ameaças representadas pela judicialização da saúde aumentando sua especialização e diferenciação. Para isso, dependerá também da resposta de outros sistemas sociais, em especial o sistema político e o próprio sistema jurídico.

A metodologia utilizada é de caráter exploratório, com realização de pesquisa bibliográfica. As informações sobre os gastos Estado do Rio Grande do Sul com a judicialização da saúde no ano de 2018 foram retiradas do site da Secretaria da Fazenda do Estado.

\section{A integridade do sistema da saúde perante as irritações do sistema jurídico}

Segundo a Teoria Geral dos Sistemas de Niklas Luhmann, a sociedade complexa evolui a partir do gerenciamento e eliminação de seus paradoxos internos. Esse processo gera a diferenciação e especialização de sistemas sociais fechados operacionalmente e abertos cognitivamente ao ambiente (LUCENA, Clarissa Santos, 2008, p. 199; ZALAZAR, Claudia; MARTINI, Sandra Regina, 2017, p. 28), a cada qual cabendo o exercício de uma função na estrutura social. Assim, o sistema sociedade engloba os demais sistemas sociais parciais (como o Direito, a Política, a Educação, a Saúde, etc.), os quais necessitam constantemente diferenciar-se do ambiente, sendo um sistema percebido como ambiente do outro (SCHWARTZ, Germano, 2018a, p. 13).

Ao sistema jurídico compete a função de manter as expectativas jurídicas ao longo do tempo, filtrando as comunicações externas através do código direito/não-direito. Nesse sentido, as Constituições são estruturas que permitem a construção de semânticas pelo sistema jurídico, sustentando a tomada de decisões jurídicas a partir do conjunto de elementos recursivos internos ao sistema (leis, jurisprudências, argumentos, etc.).

Uma característica do sistema jurídico é a imposição de condutas aos demais sistemas sociais parciais. Às frequentes irritações/perturbações provocadas pelas decisões jurídicas, os demais sistemas sociais devem responder segundo suas próprias estruturas e códigos, em um processo de constante evolução e diferenciação.

O sistema da saúde, por sua vez, não foi abordado de maneira tão aprofundada pelos teóricos da Teoria Geral dos Sistemas Sociais. Para Luhmann, esse sistema opera a partir do código saúde-doença, definindo-se a partir da função exercida pela Medicina: 
"cura delle malattie e tutto ciò che produce come sapere medico, come procedure organizzative e come forme diverse di interazione a fini di cura"3 (CORSI, Giancarlo, 2015, p. 12). Na mesma direção, SCHWARTZ, 2018b, p. 127, descreve o sistema da saúde como "un sistema autónomo y diferenciado que se autorreproduce y autoorganiza a partir de mecanismos de selectividad propios provenientes de su código binário salud/ enfermedad"4.

O sistema da saúde está em constante comunicação com os demais sistemas sociais, na medida em que as possibilidades de cuidado, tratamento e cura são dependentes de fatores como desenvolvimento científico, financiamento, regulamentações, decisões políticas e jurídicas, entre outros (CORSI, Giancarlo, 2015, p. 65; ZALAZAR, Claudia; MARTINI, Sandra Regina, 2017, p. 45). Em uma sociedade complexa, este processo resulta na evolução constante dos sistemas em respostas a novos desafios e demandas.

É a partir desta perspectiva que ZALAZAR, Claudia; MARTINI, Sandra Regina, 2017, p. 39, descrevem o processo da constitucionalização do direito à saúde no Brasil. Segundo as autoras, o reconhecimento da saúde como direito na Constituição de 1988 é consequência das irradiações sofridas pelo sistema do Direito em virtude das demandas sociais organizadas no âmbito do processo de redemocratização do país. A partir da positivação deste direito, surgem novos desafios e demandas visando sua efetivação por meio da implementação das políticas públicas de saúde - o que envolve novos conjuntos de comunicações entre os sistemas político, jurídico, econômico, da ciência, da saúde, entre outros.

O desenvolvimento tecnológico também aporta novos e constantes desafios aos sistemas sociais. Conforme salienta SCHWARTZ, Germano, 2018b, p. 127, a revolução tecnológica possibilita que a autorreprodução do sistema da saúde ocorra em um ritmo mais rápido que a de outros sistemas sociais, produzindo novas complexidades. Assim, o desenvolvimento de novos medicamentos e tratamentos geram demandas de regulamentação, financiamento e acesso, aos quais os sistemas devem responder a partir de seus próprios códigos e funções.

Para ZALAZAR, Claudia; MARTINI, Sandra Regina, 2017, p. 47, é necessário compreender o grau de autonomia e o tipo de relação entre o sistema da saúde e a totalidade dos demais sistemas sociais, bem como com cada uma de suas partes. Neste ponto, a judicialização da saúde, a partir de decisões judiciais proferidas pelo sistema jurídico, surge como um motivo de perturbação em vários sistemas.

Nesse aspecto, MARTINI, Sandra Regina, 2015, p. 50, salienta a peculiar relação do sistema de saúde com o sistema jurídico, ao descrever aquele como um "sistema social diferenciado e que constantemente recebe irritações de outros sistemas sociais, em especial - ou talvez a mais conhecida - a irritação do sistema do direito, fato definido por muitos autores como 'judicialização da saúde'". As imposições de prestação de atendimento ao sistema da saúde, de forma discricionária, pelo sistema jurídico, provocam perturbações também no sistema econômico, devido à insuficiência de

3 cura de doenças e tudo o que se produz como saber médico, como procedimentos organizacionais e como formas diversas de interação com a finalidade de curar" (tradução livre dos autores).

4 um sistema autônomo e diferenciado que se auto-reproduz e auto-organiza a partir de mecanismos de seletividade próprios provenientes de seu código binário saúde/doença" (tradução livre dos autores). 
recursos financeiros para o atendimento pleno da saúde. "Como consequência, os problemas de saúde são tratados pelo direito, fazendo com que a economia 'obrigue' a política a intervir" (MARTINI, Sandra Regina, 2015, p. 65).

Para SCHWARTZ, Germano, 2018a, p. 13, a diferenciação funcional é a base da Teoria dos Sistemas Sociais, demandando a preservação da identidade dos sistemas. "Acaso isso não ocorra, os perigos da corrupção sistêmica serão altamente prejudiciais, fazendo com que os sistemas percam sua identidade e que exista a sobreposição de um pelo outro".

A questão que se apresenta, portanto, é como as constantes perturbações provocadas pelo sistema jurídico em razão das decisões judiciais em saúde podem, de fato, representar uma excessiva interferência do sistema jurídico, comprometendo a integridade e autonomia do sistema da saúde.

Para, p, as numerosas decisões judiciais que impõem onerosos gastos com saúde representam uma ameaça ao sistema político, o qual é responsável pela implementação das políticas públicas por meio da Administração Pública, comprometendo o atendimento em saúde do conjunto da sociedade. No entender dos autores, "o direito, em sua função de manutenção das expectativas normativas, não pode - não deveria, melhor dizendo - 'interromper' a autopoiese dos demais subsistemas sociais" (LIMA, Fernando Rister de Sousa; FINCO, Matteo, 2019, p. 37).

Com o intuito de melhor compreender a maneira com que as decisões judiciais impactam o sistema da saúde, passamos a analisar este impacto a partir de informações disponíveis sobre a execução do orçamento da saúde pública pelo Estado do Rio Grande do Sul no ano de 2018.

\section{Impacto financeiro da judicialização da saúde - o caso do Estado do Rio Grande do Sul}

A judicialização da saúde vem sendo alvo de grande preocupação para pesquisadores

e gestores públicos, devido ao nível crescente de gastos do poder público com o cumprimento de decisões judiciais em saúde, em especial para a aquisição de medicamentos.

A incorporação da saúde como direito fundamental no Brasil, a partir de sua positivação na Constituição de 1988, trouxe para o sistema da saúde uma série de desafios - tanto em função da necessidade de efetivação deste direito por meio da implementação de políticas públicas quanto em decorrência da necessidade de resposta a demandas por sua efetivação pela via judicial.

Para NAUNDORF, Bruno; CARLI, Patrícia de; GOULART, Bárbara, 2018, p. 209, a judicialização da saúde no Brasil reflete a dicotomia entre a saúde coletiva e a saúde individual, na qual a organização do Sistema único de Saúde estrutura-se a partir de um viés de saúde coletiva, com ênfase na promoção da saúde e da prevenção a agravos. O conceito de saúde integral, entretanto, também garante aos cidadãos a atenção integral a doenças e agravos, por meio da disponibilização de tratamentos e instrumentos terapêuticos a partir de uma lógica individualizada. 
Nesse sentido, os grandes avanços tecnológicos, ao trazer consigo novas perspectivas para o tratamento de doenças, aportam ao sistema de saúde constantes demandas e pressões para a incorporação, regulamentação e disponibilização de novos medicamentos e demais tecnologias.

Para NAUNDORF, Bruno; CARLI, Patrícia de; GOULART, Bárbara, 2018, p. 211, a organização do atendimento à saúde é pautada pelo planejamento, e tem por base estudos e dados epidemiológicos que consideram os determinantes e condicionantes da saúde, bem como o planejamento realizado com participação social e a partir de pactuações entre os três entes federados. Nesse sentido, a judicialização excessiva termina por desorganizar o sistema, rompendo o planejamento realizado e demandando a realocação dos recursos de outras áreas.

O acesso a medicamentos e demais tratamentos de saúde pela via administrativa, idealmente, é a forma mais adequada para que essas demandas possam ser atendidas de forma planejada, organizada e sustentável, buscando a equidade e universalidade no acesso. Na prática, as demandas judiciais provocam irritações ao sistema de saúde, rompendo a lógica estabelecida nos fluxos regulares de concessão de medicamentos e demais tratamentos, competindo por recursos previstos para alocação em outras ações e prestações em saúde.

Para BUÍSSA, Leonardo; BEVILACQUA, Lucas; MOREIRA, Fernando Henrique Barbosa Borges (2018, p. 27), a judicialização da saúde acarreta distorções severas nos orçamentos públicos tanto da União quanto dos Estados e Municípios, que possuem recursos insuficientes para o atendimento à crescente demanda. Assim, em face das determinações judiciais para atendimento de demandas em saúde, o gestor usualmente termina por remanejar recursos previstos para utilização em outros serviços e atendimentos da área de saúde.

Para GEBRAN NETO, João Pedro (2014, p. 10) a judicialização da saúde agrava o problema de alocação dos já escassos recursos para o custeio da saúde pública no Brasil, sendo a busca pelo acesso a medicamentos o objeto da grande maioria das lides.

NAUNDORF, Bruno; CARLI, Patrícia de; GOULART, Bárbara, 2018, p. 210 situam no ano de 1998 o início do processo de judicialização de massa no Estado do Rio Grande do Sul, com aumento significativo e constante dos valores destinados ao pagamento de demandas judiciais até o ano de 2015. Segundo os autores, em 2016 o atendimento ao total das demandas judiciais em saúde no Rio Grande do Sul consumiu dos cofres do Estado a cifra de $\mathrm{R} \$ 275.807 .868,21$, o equivalente a $15 \%$ do total do orçamento da saúde daquele ano. Ainda segundo os autores, o impacto da judicialização do acesso a medicamentos incide tanto de forma direta, em sua aquisição, quanto de forma indireta, devido à necessidade de transporte, armazenamento, acondicionamento e distribuição dos medicamentos, bem como de realização de controle efetivo sobre sua distribuição/utilização pelo paciente, de capacitação e treinamentos constantes dos profissionais de saúde, etc.

Segundo dados disponibilizados pela Secretaria Estadual da Fazenda do Estado do Rio 
Grande do Sul ${ }^{5}$, o custo do atendimento a demandas judiciais em saúde no ano de 2018 foi de $\mathrm{R} \$ 439.729 .075,11$. Este montante representa $12,4 \%$ do total de recursos executados no período pela Secretaria Estadual de Saúde ${ }^{6}$ para financiamento e co-financiamento estadual de todas as políticas de saúde no Estado: Atenção Básica; Atenção em Saúde Mental; co-financiamento da Farmácia Básica municipal; Rede de Urgência e Emergência; co-financiamento hospitalar; Vigilância em Saúde; parcela estadual para a assistência hospitalar e ambulatorial; atenção especializada; atendimento em ambulatórios e hospitais próprios; entre outros.

Esses mesmos dados demonstram que mais de $91 \%$ dos valores despendidos com o atendimento de demandas judiciais em saúde neste período, ou o montante de $\mathrm{R} \$$ 400.713.271,95, foi utilizado para a compra de medicamentos, o que representa mais de $11 \%$ da execução orçamentária da Secretaria Estadual da Saúde no exercício de 2018 (excluídas despesas com pessoal e encargos sociais), mais que o triplo do valor dispendido pelo Estado do Rio Grande do Sul com a compra dos medicamentos fornecidos administrativamente ${ }^{7}$, e quase $150 \%$ do total do valor investido pelo Estado para o co-financiamento da Atenção Básica.

\section{Judicialização da saúde no Rio Grande do Sul e a integridade do sistema da saúde} Os dados referentes às despesas do Estado do Rio Grande do Sul com a judicialização da saúde demonstram o significativo impacto orçamentário exercido pelas decisões judiciais em saúde, em especial pelas demandas por acesso a medicamentos. Tratase de valores significativos, que acarretam desequilíbrios no sistema da saúde ao desconsiderar sua estrutura e funcionamento internos, determinando necessária realocação dos recursos finitos de que dispõe.

Não por menos, GEBRAN NETO, João Pedro (2014, p. 27) critica veementemente o papel do excesso de interferências do Poder Judiciário que, através de suas decisões, termina por tornar-se de fato um ordenador de despesas e políticas públicas, sem possuir legitimidade para tal. Também segundo o jurista, a excessiva intervenção do Poder Judiciário termina por promover uma iníqua distribuição de recursos em detrimento das políticas postas.

O conjunto de argumentos aportados sugere que, no limite, o grande número de decisões judiciais contra o Estado pode gerar uma corrupção do sistema da saúde, comprometendo o atendimento em saúde do conjunto da sociedade. Diante desta situação, o sistema deve responder buscando ampliar sua diferenciação. A integridade do sistema da saúde diante dos riscos aportados pela judicialização depende de "um aumento na sofisticação da legislação em matéria de saúde e, portanto, da complexidade que o sistema é capaz de lidar (limitando) a influência que ele pode sofrer de outros sistemas, em particular do direito" (LIMA, Fernando Rister de Sousa e FINCO, Matteo, 2019, p. 38).

\footnotetext{
5 Fonte: site da Secretaria da Fazenda do Estado do Rio Grande do Sul. Disponível em: https://www.sefaz.rs.gov.br/AFE/DOT-DES_1.aspx. Acesso em: 10 de jun. de 2019.

6 Execução orçamentária da Secretaria Estadual de Saúde incluindo despesas correntes e investimentos, excluídas despesas com pessoal e encargos sociais. Fonte: site da Secretaria da Fazenda do Estado do Rio Grande do Sul. Disponível em: https://www.sefaz.rs.gov.br/AFE/DOT-DES_1.aspx. Acesso em: 10 de jun. de 2019

7 No ano de 2018, o gasto do Estado com a compra de medicamentos fornecidos por via administrativa foi de R\$ 111.509.005,60. Disponível em: https://www. sefaz.rs.gov.br/AFE/DOT-DES_1.aspx. Acesso em: 10 de jun. de 2019.

8 No período, o financiamento do Estado para a Atenção Básica correspondeu a R\$ 266.800.431,18. Disponível em: https://www.sefaz.rs.gov.br/AFE/DOTDES_1.aspx. Acesso em: 10 de jun. de 2019.
} 
Assim, conforme os pressupostos da Teoria Geral dos Sistemas, o caminho para a manutenção da integridade do sistema da saúde passa por sua capacidade de resposta às perturbações advindas do sistema jurídico, uma vez que este seguirá produzindo decisões, em conformidade com a função que lhe cabe, de acordo com sua própria estrutura e código internos. Isso dependerá também de respostas dos demais sistemas sociais também afetados (em especial o econômico e o político).

A título de exemplo, a Emenda Constitucional 29/20009 representou um avanço para a segurança do financiamento da saúde pública no país, determinando transparência e garantia de alocação de recursos destinados às ações e políticas em saúde. Estes recursos, entretanto, permanecem insuficientes para o atendimento do conjunto das demandas em saúde, o que demanda uma organização interna do sistema no sentido de definir prioridades e critérios claros para a alocação destes recursos.

Em relação à estruturação do sistema visando garantir o acesso da população a novas tecnologias e medicamentos de maneira segura, organizada e sustentável, destacam-se como importantes avanços a Lei $n^{\circ} 12401 / 2011^{10}$ e a Lei $n^{0} 13.411 / 2016^{11}$, que definiram fluxos, processos e prazos para a incorporação de novas tecnologias em saúde no âmbito do SUS. Estas leis funcionam como novas comunicações para os diferentes sistemas sociais, em especial os sistemas da saúde, jurídico, econômico e o próprio sistema político do qual elas são fruto.

Exemplo do impacto exercido por estas iniciativas é a recente decisão do Plenário do Supremo Tribunal Federal, que originou o Tema 500 de Repercussão Geral ${ }^{12}$, fundamentada principalmente na Lei $n^{\circ}$ 13.411/2016 e que estabelece a necessidade de atendimento aos fluxos e limites desta Lei para a solicitação de acesso a medicamentos pela via judicial.

A referida Tese de Repercussão Geral impacta o sistema da saúde ao limitar a concessão judicial de medicamentos que não possuam registro sanitário quando não for demonstrada mora irrazoável da Anvisa na apreciação do pedido. SCHULZE, Clenio Jair (2019), em breve comentário sobre a decisão, destaca seus efeitos positivos ao conjunto da sociedade, na medida em que reforça a não somente a responsabilidade da Agência Nacional de Vigilância Sanitária na análise de registros de novos medicamentos, como também a necessidade de adequação das condutas dos próprios laboratórios

\footnotetext{
9 Emenda Constitucional no 29, de 13 de setembro de 2000. Altera os arts. 34, 35, 156, 160, 167 e 198 da Constituição Federal e acrescenta artigo ao Ato das Disposições Constitucionais Transitórias, para assegurar os recursos mínimos para o financiamento das ações e serviços públicos de saúde. Disponível em: http://www.planalto.gov.br/ccivil_03/constituicao/emendas/emc/emc29.htm. Acesso em: 11 de jun. de 2019.

10 Lei $n^{\circ}$ 12.401, de 28 de abril de 2011. Altera a Lei no 8.080, de 19 de setembro de 1990, para dispor sobre a assistência terapêutica e a incorporação de tecnologia em saúde no âmbito do Sistema Único de Saúde - SUS. Disponível em: http://www.planalto.gov.br/ccivil_03/_ato2011-2014/2011/lei/112401.htm. Acesso em: 11 de jun. de 2019.

11 Lei $\mathrm{n}^{\circ}$ 13.411, de 28 de dezembro de 2016. Altera a Lei $\mathrm{n}^{\circ}$ 6.360, de 23 de setembro de 1976, que dispõe sobre a vigilância sanitária a que ficam sujeitos os medicamentos, as drogas, os insumos farmacêuticos e correlatos, cosméticos, saneantes e outros produtos, e dá outras providências, e a Lei nº 9.782 , de 26 de janeiro de 1999, que define o Sistema Nacional de Vigilância Sanitária, cria a Agência Nacional de Vigilância Sanitária, e dá outras providências, para dar transparência e previsibilidade ao processo de concessão e renovação de registro de medicamento e de alteração pós-registro. Disponível em: https://www2. camara.leg.br/legin/fed/lei/2016/lei-13411-28-dezembro-2016-784151-publicacaooriginal-151761-pl.html. Acesso em: 11 de jun. de 2019.

12 Tese de Repercussão Geral: "1. O Estado não pode ser obrigado a fornecer medicamentos experimentais. 2. A ausência de registro na ANVISA impede, como regra geral, o fornecimento de medicamento por decisão judicial. 3. É possível, excepcionalmente, a concessão judicial de medicamento sem registro sanitário, em caso de mora irrazoável da ANVISA em apreciar o pedido (prazo superior ao previsto na Lei no $13.411 / 2016$ ), quando preenchidos três requisitos: (i) a existência de pedido de registro do medicamento no Brasil (salvo no caso de medicamentos órfãos para doenças raras e ultrarraras); (ii) a existência de registro do medicamento em renomadas agências de regulação no exterior; e (iii) a inexistência de substituto terapêutico com registro no Brasil. 4. As ações que demandem fornecimento de medicamentos sem registro na ANVISA deverão necessariamente ser propostas em face da União". Disponível em: <http://stf.jus. br/portal/jurisprudenciaRepercussao/abrirTemasComRG.asp >. Acesso em: 11 de jun. de 2019.
} 
farmacêuticos, os quais utilizavam-se da estratégia da judicialização para subverter a lógica da regulação sanitária brasileira.

Estima-se que o rápido avanço das tecnologias na área da saúde seguirá trazendo consigo novas demandas, que serão percebidas como irritações sistêmicas, trazendo novos desafios ao sistema da saúde. A capacidade de o conjunto dos sistemas sociais responderem a estas perturbações, aumentando sua especialização, definirá a possibilidade de manutenção de sua integridade e diferenciação.

\section{Considerações finais}

No contexto da Teoria Geral dos Sistemas Sociais de Niklas Luhmann, uma característica do sistema jurídico é a imposição de conduta aos demais sistemas sociais parciais, por meio das decisões judiciais por ele proferidas. Essas decisões são sentidas pelos demais sistemas como irritações as quais, no limite, podem provocar uma corrupção sistêmica. Por outro lado, as respostas dos diferentes sistemas sociais a essas irritações também podem provocar reações que visem o aumento da diferenciação sistêmica, ocasionando sua evolução.

Dados referentes à execução orçamentária do Governo do Estado do Rio Grande do Sul demonstram o risco de corrupção sistêmica ocasionado pela judicialização da saúde, na medida em que, no ano de 2018, mais de $12 \%$ do orçamento da Secretaria Estadual da Saúde foi destinado ao atendimento de decisões judiciais, em sua grande maioria visando à dispensação de medicamentos. Esses recursos devem ser realocados de outras fontes e rubricas e, no limite, podem comprometer e inviabilizar a própria efetivação do direito à saúde por meio da implementação e financiamento do conjunto de programas e ações componentes da política pública de saúde.

A integridade do sistema da saúde diante dos desafios aportados pelo fenômeno da judicialização da saúde depende da capacidade de resposta estruturada dos demais sistemas sociais, em especial do sistema Político e do sistema Econômico. Nesse sentido, no Brasil, diferentes respostas já foram estruturadas, como a aprovação de um mínimo constitucional para investimento em saúde e a aprovação de leis que aprimoram os fluxos e condições para incorporação de novas tecnologias de saúde e para o acesso a estas tecnologias.

Caberá não somente ao sistema da saúde, mas ao conjunto dos sistemas sociais, responder às irritações sistêmicas provocadas pela judicialização da saúde. Assim, a capacidade de o conjunto dos sistemas sociais responderem a estas irritações, aumentando sua especialização, definirá a possibilidade de manutenção de sua integridade e diferenciação. 


\section{REFERÊNCIAS BIBLIOGRÁFICAS}

- BRASIL. Supremo Tribunal Federal (2019): Recurso Extraordinário 657718. Relator Min. Marco Aurélio, Julgamento em: 23 Mai. 2019.

- BUÍSSA, Leonardo; BEVILACQUA, Lucas; MOREIRA, Fernando Henrique Barbosa Borges (2018): "Impactos Orçamentários da Judicialização das Políticas Públicas de Saúde", em SANTOS, Alethele de Oliveira; LOPES, Luciana Tolêdo: Coletânea direito à saúde: dilemas do fenômeno da judicialização da saúde, CONASS, Brasília - DF, pp. 26-50.

- CONSELHO NACIONAL DE JUSTIÇA - CNJ (2019): Judicialização da Saúde no Brasil: perfil das demandas, causas e propostas de solução, CNJ, Brasília.

- CORSI, Giancarlo (2015): Salute e malattia nella teoria dei sistemi. A partire da Niklas Luhmann, Franco Angeli, Milão.

- GEBRAN NETO, João Pedro (2014): Direito à Saúde - Direito constitucional à saúde e suas molduras jurídicas e fáticas. Disponível em: https://www.conass.org.br/consensus/ wp-content/uploads/2015/04/Artigo-direito-a-saude.pdf, acesso em: 10.06.2019.

- LIMA, Fernando Rister de Sousa; FINCO, Matteo (2019): “Há Limite Econômico para a Intervenção do Judiciário na Saúde Pública?", em RBSD - Revista Brasileira de Sociologia do Direito, Vol. 6, Nro. 1, pp. 27-41.

- LUCENA, Clarissa Santos (2008): “Perspectivas Autopoiéticas para a Função Social dos Contratos" em TUTIKIAN, Cristiano: Olhares sobre o Público e o Privado, EDIPUCRS, Porto Alegre.

- LUHMANN, Niklas (2000): “O Paradoxo dos Direitos Humanos e Três Formas de seu Desdobramento", em Themis, Vol. 3, Nro. 1, Fortaleza, pp. 153-161.

- LUHMANN. Niklas (2016): Sistemas Sociais: esboço de uma teoria geral. Vozes, São Paulo. - MARTINI, Sandra Regina (2015): "Sistema de Saúde e Transformação Social", em MARTINI, Sandra Regina, Saúde, Direito e Transformação Social: um Estudo sobre o direito à saúde no Rio Grande do Sul. Livraria do Advogado Editora, Porto Alegre.

- NAUNDORF, Bruno; CARLI, Patrícia de; GOULART, Bárbara (2018): “O Estado do Rio Grande do Sul e os Impactos da Judicialização da Saúde na Gestão Pública”, em SANTOS, Alethele de Oliveira; LOPES, Luciana Tolêdo: Coletânea direito à saúde: dilemas do fenômeno da judicialização da saúde, CONASS, Brasília - DF, pp. 208-217.

- NEVES, Rômulo Figueira (2005): Acoplamento Estrutural, Fechamento Operacional e Processos Sobrecomunicativos na Teoria dos Sistemas Sociais de Niklas Luhmann. Dissertação (Mestrado em Sociologia) - Faculdade de Filosofia, Letras e Ciências Humanas da Universidade de São Paulo, São Paulo.

- SCHWARTZ, Germano (2018a): As Constituições Estão Mortas? Momentos Constituintes e Comunicações Constitucionalizantes dos Novos Movimentos Sociais do Século XXI. Lumen Juris, Rio de Janeiro.

- SCHWARTZ, Germano (2018b): “Lo Humano y los Humanos de los Dereches Humanos. Animales, Pacha Mama y Alta Tecnología", em Economía y Política 5(I), pp. 113-139.

- SCHULZE, Clenio Jair (2019): STF, Registro na Anvisa e Judicialização da Saúde. Tomado de: https://emporiododireito.com.br/leitura/stf-registro-na-anvisa-e-judicializacao-dasaude, acesso em: 11/06/2019.

- ZALAZAR, Claudia; MARTINI, Sandra Regina (2017): Sociología, Metodología, Derecho y Salud em la Sociedad Compleja, Advocatus, Córdoba. 